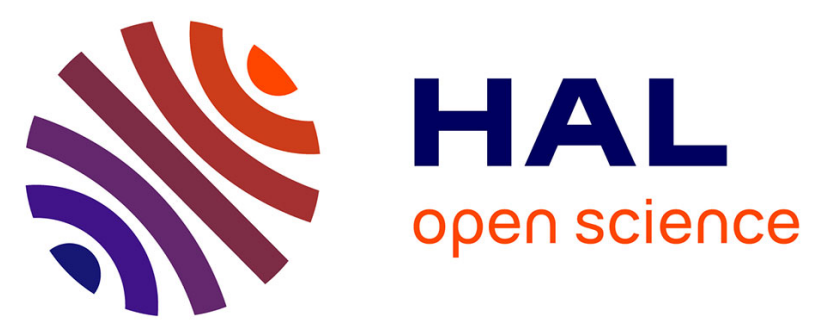

\title{
Magnetocaloric Effect in Flexible, Free-Standing Gadolinium Thick Films for Energy Conversion Applications
}

Doan Nguyen Ba, Yunlin Zheng, Loic Becerra, Massimiliano Marangolo, Morgan Almanza, Martino Lobue

\section{To cite this version:}

Doan Nguyen Ba, Yunlin Zheng, Loic Becerra, Massimiliano Marangolo, Morgan Almanza, et al.. Magnetocaloric Effect in Flexible, Free-Standing Gadolinium Thick Films for Energy Conversion Applications. Physical Review Applied, 2021, 15 (6), 10.1103/PhysRevApplied.15.064045 . hal03264108

\section{HAL Id: hal-03264108 \\ https://hal.science/hal-03264108}

Submitted on 18 Jun 2021

HAL is a multi-disciplinary open access archive for the deposit and dissemination of scientific research documents, whether they are published or not. The documents may come from teaching and research institutions in France or abroad, or from public or private research centers.
L'archive ouverte pluridisciplinaire HAL, est destinée au dépôt et à la diffusion de documents scientifiques de niveau recherche, publiés ou non, émanant des établissements d'enseignement et de recherche français ou étrangers, des laboratoires publics ou privés. 


\title{
Magnetocaloric Effect in Flexible, Free-Standing Gadolinium Thick Films for Energy Conversion Applications
}

\author{
Doan Nguyen Ba $\odot,{ }^{1,2,}{ }^{*}$ Yunlin Zheng $\odot,{ }^{2}$ Loic Becerra, ${ }^{2}$ Massimiliano Marangolo $\odot,{ }^{2, \dagger}$ \\ Morgan Almanza $\odot, 1, \ddagger$ and Martino LoBue $\odot 1, \S$ \\ ${ }^{1}$ Université Paris-Saclay, ENS Paris-Saclay, CNRS, SATIE, 91190 Gif-sur-Yvette, France \\ ${ }^{2}$ Sorbonne Université, CNRS, Institut des NanoSciences de Paris, UMR7588, F-75252 Paris, France
}

(Received 3 March 2021; revised 26 April 2021; accepted 7 June 2021; published 17 June 2021)

\begin{abstract}
This study presents a method of producing thick (i.e., in the $\mu \mathrm{m}$ range) polycrystalline Gd free-standing flexible films. Preparation is carried out by sputtering on silicon conventional substrates using tantalum as a buffer and capping layer. Magnetic and magnetocaloric properties show good agreement with data from high-purity bulk $\mathrm{Gd}$, and are not altered by substrate removal. Moreover, the free-standing film is flexible and all the relevant magnetic properties (i.e., Curie temperature $T_{c}$, saturation magnetization $M_{s}$, and isothermal entropy change $\Delta S$ ) are preserved under bending (up to a $\varepsilon= \pm 0.78 \%$ strain over the two film sides). The technological opportunities heralded by availability of magnetocaloric flexible self-sustaining films are discussed in the conclusions with particular focus on energy-conversion applications (i.e., cooling and thermal energy harvesting). More precisely, the output-power upper bound of an thermal-energy harvester deploying a Gd flexible film with the reported properties is worked-out using a thermal switch model presented elsewhere. The calculations show a potential output able to supply the new generation of IoT wireless devices as well as small medical implants. The result moot Gd free-standing flexible films as a benchmark for a new generation of small high-throughput magnetocaloric energy-conversion devices.
\end{abstract}

DOI: 10.1103/PhysRevApplied.15.064045

\section{INTRODUCTION}

For the last 20 years, the research into high-magneticmoment films for microscale and nanoscale applications has been led by the need for large magnetic fields at the small scale, aiming technologies ranging from magnetic resonance imaging, to information storage [1]. The scope of these investigations has been remarkably widened in association with the use of magnetic free-standing films and membranes as components in microelectromechanical systems (MEMS), as permanent magnets [2,3], and more recently as active substances for energy conversion (i.e., cooling, heat pumps, and thermal-energy harvesters) [4-6]. In addition to the need to preserve the exchange energy (i.e., the Curie temperature $T_{c}$ ), and the magnetic moment per unit cell (i.e., the saturation magnetization $M_{s}$ ) of the bulk material, the latter applications require excellent magnetocaloric (MC) properties (i.e., high isothermal entropy change $\Delta S$, and adiabatic temperature change $\Delta T_{\text {adia }}$ ) [4,7], and high thermal diffusivity (i.e., to foster

\footnotetext{
*badoan2309@gmail.com

†massimiliano.marangolo@insp.jussieu.fr

†morgan.almanza@ens-paris-saclay.fr

§Martino.Lo-bue@ens-paris-saclay.fr
}

thermal contact with other components or with heat fluids). In this regard, the research on free-standing films represents a key task towards suitable application of MC materials into energy-conversion microdevices.

In addition, microfabrication techniques and device working conditions impose further specifications so that easy-to-handle and flexible films that can be twisted without losing their functional properties have become highly regarded for applications in medical implants and wearable devices $[8,9]$.

Gadolinium, with its $7.6 \mu_{B}$ per atom, and with a close to room-temperature Curie point $T_{c} \approx 294 \mathrm{~K}[10,11]$, has been recognized as a promising single-element magnetic material for low-temperature high-magnetic-moment applications [12,13]. Moreover, bulk Gd has been the benchmark material for MC properties [7], and Gd films will arguably follow a similar path in the domain of microscale and nanoscale energy-conversion applications $[14,15]$. Getting thick (in the micrometer range), freestanding Gd films can be particularly relevant for energytransduction applications where achieving high conversion powers, and avoiding the reduction in caloric properties associated with the buffer heat capacity are the key objectives. So far, most of the existing literature has been devoted either to films in the thickness interval 5-100 $\mathrm{nm}$ [16] or to melt-spun [17-19] and cold-rolled [20-22] 
ribbons. The former are definitely too thin to get the powers needed for energy-conversion applications, the latter got some rather interesting properties in the thickness range 20-200 $\mu \mathrm{m}$. However cold-rolling and meltspinning both require further annealing to partially recover the bulk properties. In addition to flexibility and surface characterization, two key properties as we argue in what follows, have seldom been made available for ribbons and thin films either. Let us consider, as an example, the $120-\mu \mathrm{m}$-thick film fabricated using a vacuum induction evaporation method on Hastelloy substrate presented in Ref. [23]. This thick free-standing film has been designed to optimize heat exchange with a fluid (i.e., aiming a regenerator device) and it shows a rather irregular dimplelike surface structure with domes as high as $530 \mu \mathrm{m}$. This hills and valleys surface is expected to offer a major hindrance to the solid-solid thermal contact we aim for here, and flexibility, if any, has not been presented.

Here we report the production of textured, flexible, freestanding polycrystalline Gd film of thickness $h=17 \mu \mathrm{m}$. The magnetic, and MC properties are measured before and after substrate removal, and their stability tested against bending (i.e., under strain). In addition, the choice of the capping layers, the deposition conditions, and the separation from the substrate process have been carried to obtain a high-quality smooth surface on both sides, suitable for applications where heat exchange takes place through solid-solid direct contact.

\section{EXPERIMENTAL DETAILS}

\section{A. Film deposition}

Gd films have been deposited on silicon (100) substrates with a 290-nm thermally oxidized layer (Si (100)/ $/ \mathrm{SiO}_{2} 290 \mathrm{~nm}$ ) by DC sputtering in an argon atmosphere. Deposition has been carried out at an approximately $3 \mu \mathrm{m} / \mathrm{h}$ rate on a substrate heated above room temperature $(\sim 543 \mathrm{~K})$. The $85 \mathrm{~mm}$ diameter, $99.9 \% \mathrm{Gd}$ target, was placed at $65 \mathrm{~mm}$ from the sample holder. Base pressure was approximately $1 \times 10^{-7}$ mbar at the depositing temperature. Tantalum (chosen because of its corrosion resistance, flexibility, and high thermal conductivity) deposited by DC sputtering under the same condition as $\mathrm{Gd}$, has been used as the buffer and capping layer. Silicon substrate surface was cleaned by a sputtering system which removed approximately $30 \mathrm{~nm}$ of the $\mathrm{SiO}_{2}$ layer (checked by a scanning electron microscope).

\section{B. Preparation of the free-standing films}

In order to pull out the Gd layer from the substrate, we pushed the film against a convex surface after performing a superficial cut on the free side of the silicon substrate as shown in Fig. 1. This allows the substrate to be broken without damaging the $\mathrm{Gd}$ film. The thermomagnetic

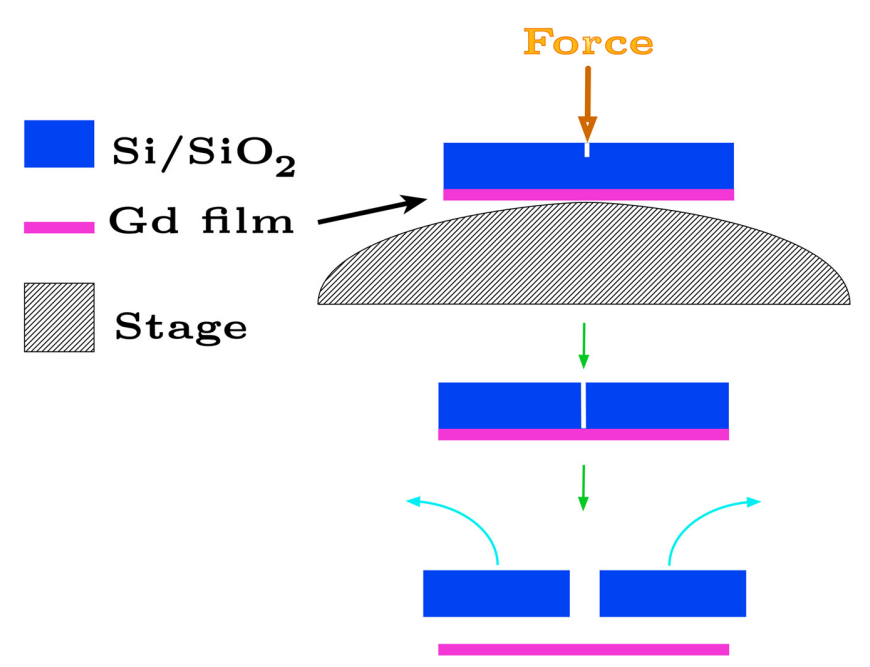

FIG. 1. Schematic for the separation of the $\mathrm{Si} / \mathrm{SiO}_{2}$ substrate from the Gd film.

curves before and after peeling off show no differences (see the inset in Fig. 6), and the film is not scrolled after removing the substrate. The free-standing film has been produced with a cleft close to the edge of the sample, as shown in Fig. 2, and then reduced from its original size of $10 \times 20 \mathrm{~mm}^{2}$ by cutting it down to $2.5 \times 2.5 \mathrm{~mm}^{2}$ using scissors.

It is worth noting that thickness, depositing temperature, and the buffer layer choice are key elements to obtain an undamaged free-standing film using the technique described here. For instance, the peeling-off process can result in film damage for thicknesses less than $5 \mu \mathrm{m}$, and for films deposited at room temperature. In addition, Gd films teared after the detaching process when using tungsten as a capping layer.

It turns out that there are critical thicknesses, optimal depositing temperatures as well as preferred buffer layers

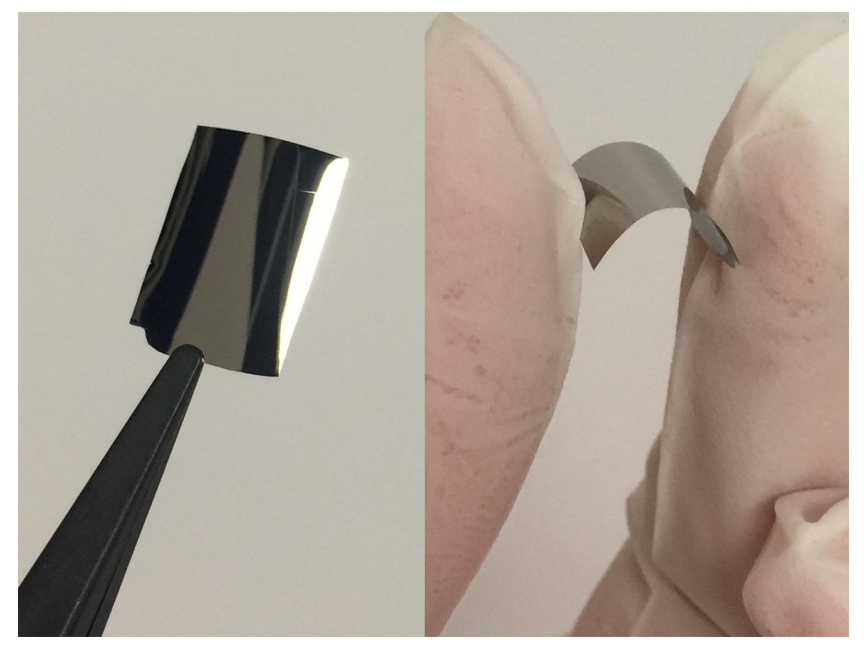

FIG. 2. Photo of the Gd free-standing film. 
in order to get high-quality free-standing films. A systematic study on the relevance of deposition temperature will be presented elsewhere.

Interestingly, our Gd free-standing films can be bent as shown in Fig. 2 and released recovering their original shape without any apparent difference and without detrimental changes in the magnetic properties. This shows that the film is easy to handle, a pretty relevant property to integrate the film into a MEMS device through microfabrication techniques.

\section{Microstructure}

A cross-section image of the free-standing film after cutting is shown in Fig. 3. The film looks dense and continuous, without porosity. The apparent columnar structure has been previously reported in Gd thick films [14], and can be related to the high deposition rate, and to similar structures observed in the cross-section of the tantalum layer (not shown here).

We probed the thickness of the free-standing film along its length. The measured thickness by field emission scanning electron microscopy (FESEM) is $h \approx 17 \mu \mathrm{m}$. Cutting with scissors can introduce local compressive stresses or expanded rough points along the sample edges. In addition, the uncut film shows an approximately 5\% thickness variation over $10 \mathrm{~mm}$ from the center to the edges. Nonetheless, this effect is negligible in the cut $2.5 \times 2.5 \mathrm{~mm}^{2}$ sample used for magnetic characterizations.

X-ray diffraction (XRD) patterns of the film are shown in Fig. 4. Owing to the film thickness, $\mathrm{X}$-rays cannot probe the whole film so that we performed XRD on both film sides. We refer to the side previously in contact with the substrate as the bottom and to the other as the top.

The high intensity of the (110) peak of the top XRD pattern reveals a preferred texturing along this direction

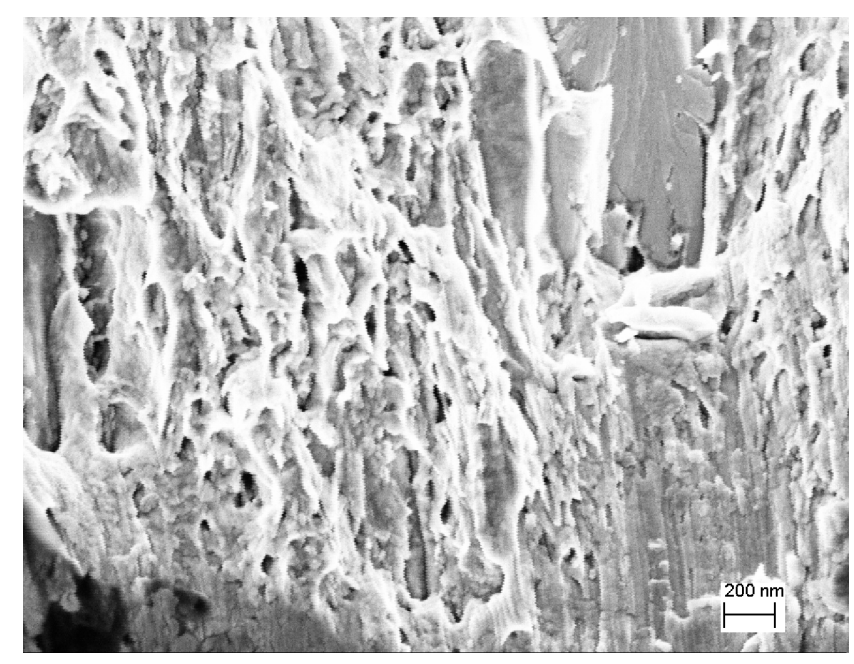

FIG. 3. In-lens cross-section of the free-standing film.

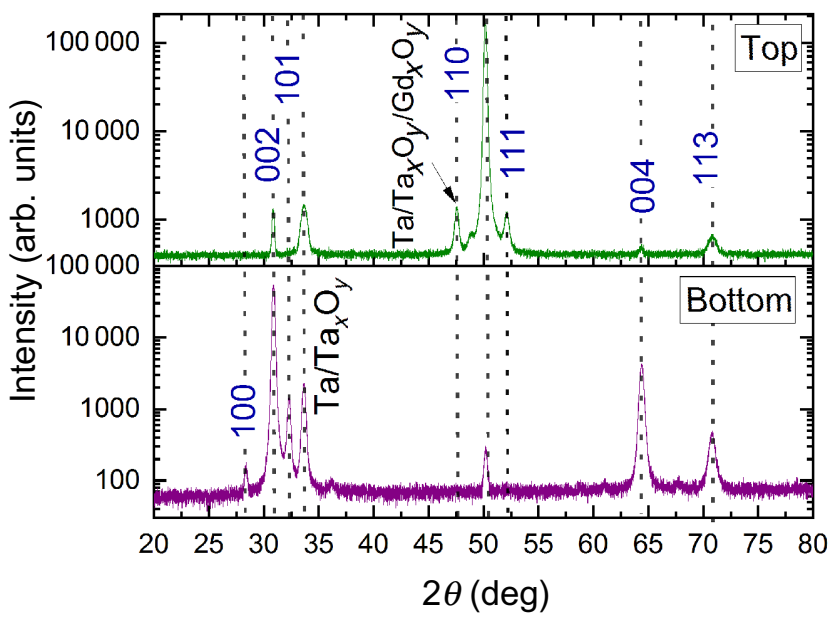

FIG. 4. XRD patterns for the top and bottom of the film.

(i.e., $c$ is preferentially laying in the film plane). Through a $\log _{10}$-linear plot, we can detect other peaks of $\mathrm{Gd}$, Ta, or $\mathrm{Ta}_{2} \mathrm{O}_{5}$. However, tantalum-related peaks are very weak and, showing only one reflection, they cannot be indexed. Relative intensity of other peaks is less than $0.5 \%$ in the top part of the film. Crystallite sizes of the (110) and (002) are 54 and $41 \mathrm{~nm}$, respectively. We observed additional peaks at $2 \theta=47.542^{\circ}$, which may point at the presence of a very small amount of tantalum or gadolinium oxide. Measurements using Rutherford backscattering spectrometry (RBS) show the presence of a small amount of gadolinium oxide. Actually, using peak integrals the amount of gadolinium oxide is estimated at approximately $0.8 \%$. Table I summarizes peak relative intensities and crystallite sizes extracted through Scherrer formula for the top and bottom films surfaces.

The bottom XRD pattern shows a rather different texturing with (001) preferential orientation (i.e., $c$ is preferentially perpendicular to the film plane). The main reflection 002 is high and narrow whereas the other peaks intensities are pretty weak (see Table I for a summary). No traces of Gd oxide are observed. It is worth noting that the Ta capping layer is $91.5 \mathrm{~nm}$ on the bottom side and $15.35 \mathrm{~nm}$ on the top.

We estimated the lattice parameter of the free-standing film with the $d$-spacing $d_{110}=1.81677 \AA$, $a=3.6335 \AA$.

TABLE I. Crystallite sizes in nanometer and relative intensities of the free-standing film. Crystallite size is estimated using the Scherrer formula.

\begin{tabular}{lccccccc}
\hline \hline & 100 & 002 & 101 & 110 & 111 & 004 & 113 \\
\hline Top (nm) & & 41 & & 54 & 18 & 35 & 13 \\
Relative intensity (\%) & & 0.5 & & 100 & 0.4 & 0.04 & 0.12 \\
Bottom (nm) & 26 & 40 & 32 & 41 & & 29 & 16 \\
Relative intensity (\%) & 0.1 & 100 & 2.5 & 0.4 & & 8.2 & 0.7 \\
\hline \hline
\end{tabular}


From the literature [11], Gd high-purity sample (99.8\%) shows $a=3.6336 \AA$ and $c=5.7810 \AA$. This good agreement suggests good crystallinity and the absence of residual stress.

In short, XRD pattern of both film sides suggests a highquality film. The main difference between the two sides is the texturing, with a (110) and (001) preferred orientation on the top and on the bottom, respectively.

\section{Surface morphology}

Smoothness, jointly with flexibility, are key features to develop caloric materials applications at the microscale where heat flux has to take place mostly through solid-solid direct thermal contact, rather than through a fluid exchange substance [24,25]. Indeed, surface roughness can hinder heat exchange, limiting the device working frequency (i.e., the power).

The flexible film we study here reflects like a mirror, as shown in Fig. 2, a property hinting at a good surface quality, that is, smoothness. For further investigation, we scanned the top and the bottom film surface by FESEM (Fig. 5) and probed them using atomic force microscopy (AFM) with a Park Systems NX20 model.

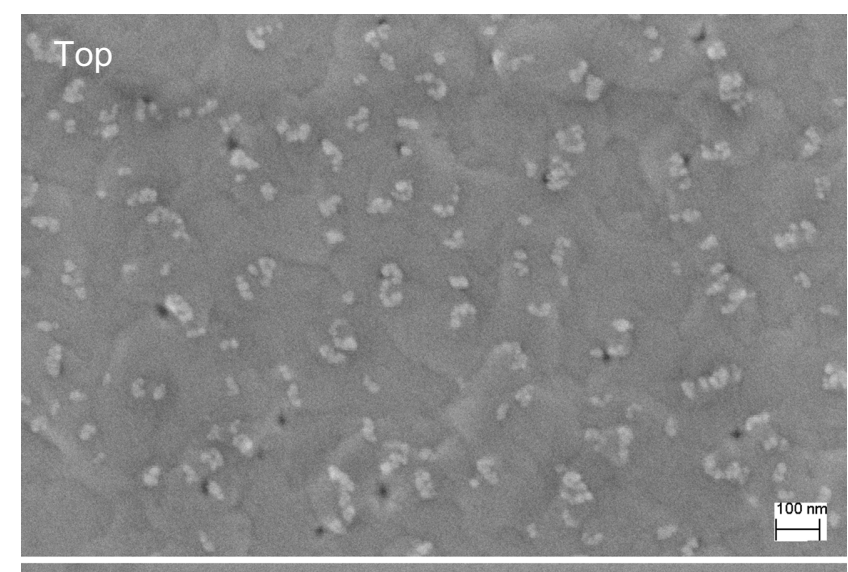

Bottom

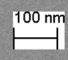

FIG. 5. Surface morphology of flexible films scanned by FESEM.
FESEM images of the top and bottom film surfaces are shown in Fig. 5. Both surfaces look smooth and no cracks can be appreciated. Grains of size up to $200 \mathrm{~nm}$ are apparent on the top surface, whereas the bottom looks more uniform. The observed difference can be related to the role of the substrate, in contact with the bottom side during the growth process, to the film texturing ( $c$ axis is mostly perpendicular to the bottom, and parallel to the top surface favoring the arabesque grain pattern apparent on the latter), and to the thicker capping layer present on the bottom.

AFM probing gives a quantitative estimation of the profile roughness. The arithmetical mean deviation $R_{a}$, and the root mean squared $R_{\mathrm{RMS}}$ roughness are $R_{a} \sim 8-11 \mathrm{~nm}$ and $R_{\mathrm{RMS}} \sim 10-14.5 \mathrm{~nm}$ for the top and $R_{a} \sim 1.6-4.8 \mathrm{~nm}$ and $R_{\mathrm{RMS}} \sim 2.4-6.3 \mathrm{~nm}$ for the bottom. Notwithstanding the slight difference between the film sides, here again to be ascribed to the texturing and to the capping layer thickness, it is worth noting that a roughness $R_{\mathrm{RMS}}\left(R_{a}\right)$ less than 15 (11) $\mathrm{nm}$ will not hinder the thermal contact with the heat reservoirs.

\section{E. Magnetic properties}

All the magnetic measurements have been performed by applying the field parallel to the film plane in order to obtain negligible demagnetizing effects (i.e., the applied and the internal field coincide) due to the film geometry. Thermomagnetic curves $M(T)$ have been recorded at fixed fields $H$ (i.e., $H=0.05,1,3$, and $5 \mathrm{~T}$ ) using a Quantum Design MPMS XL. Isothermal magnetization curves have been measured using a Quantum Design PPMS applying a field ramp spanning from $H_{i}=0$ up to $H_{f}=7 \mathrm{~T}$. Hysteresis magnetization curves have been measured at different temperatures below the Curie point.

\section{Magnetization as a function of temperature}

Thermomagnetic $M(T)$ measurements on the freestanding film under different fields are shown in Fig. 6, whereas in the inset the free-standing film $M(T)$ curve under $1 \mathrm{~T}$ applied field is compared with the same measurement carried on the Gd film before substrate removal. No relevant differences can be appreciated confirming that neither the magnetization, nor the Curie temperature have been altered by the peeling-off process.

The Curie temperature of the free-standing film has been extracted from the thermomagnetic measurements both using the vanishing magnetization point under small applied field (i.e., $H=0.001 \mathrm{~T}$ ) and through the first derivative of $M(T)$ under $H=0.05 \mathrm{~T}$; both curves are shown in the main frame of Fig. 6. Both extrapolations give $T_{c}=293 \pm 3 \mathrm{~K}$ in good agreement with the reported bulk values [26]. Working out $T_{c}$ using the inflection point method [27] gives a slightly higher value, $T_{c}=299.5 \pm 2.5 \mathrm{~K}$ (see Table II for a summary of Curie 


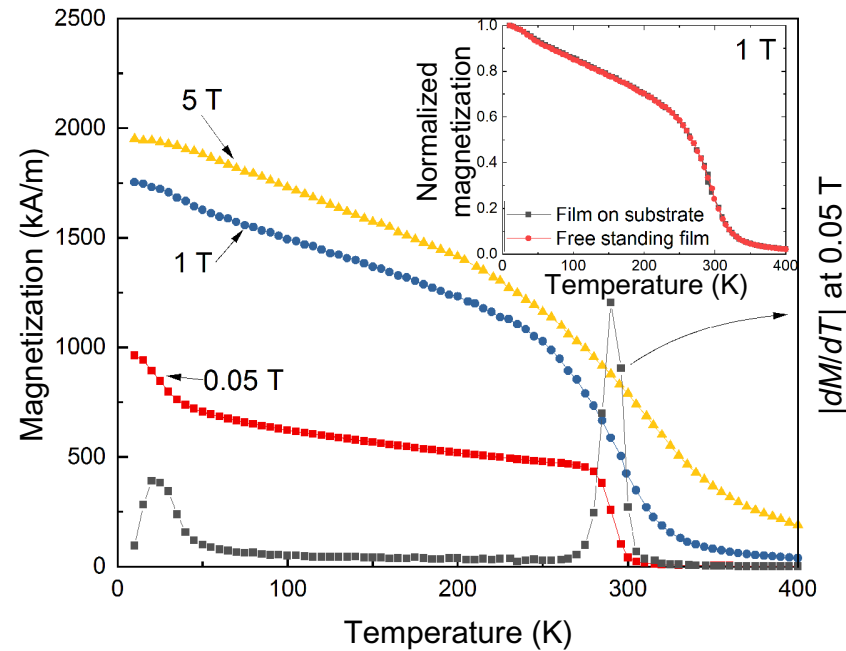

FIG. 6. Main frame: $M(T)$ curves under different applied magnetic fields measured on the free-standing film (left axis) and $|d M(T) / d T|$ at $H=0.05 \mathrm{~T}$ (right axis). Inset: comparison between the thermomagnetic curves, $M(T)$, measured on the free-standing film and on the film still on the substrate (i.e., before peeling off) under an applied field of $1 \mathrm{~T}$.

temperature values determined through different techniques).

The maximum magnetization at $10 \mathrm{~K}$ under an applied field $H=5 \mathrm{~T}$ is $M=1951 \mathrm{kA} / \mathrm{m}$ in good agreement with the bulk value reported in the literature [26] and with our measurement on thick films grown on $\mathrm{Si} / \mathrm{SiO}_{2}$ substrates [14].

The magnetization bump appearing on $M(T)$ under weak applied field below $25 \mathrm{~K}$ has been reported and discussed in the literature as well as in our previous study [14].

\section{Isothermal magnetization measurements}

Isothermal magnetization curves have been measured from 400 to $10 \mathrm{~K}$ under application of a magnetic field ramp from $H_{i}=0 \mathrm{~T}$ up to $H_{f}=7 \mathrm{~T}$. Results are shown

TABLE II. Determination of the Curie temperature of the freestanding Gd film by different techniques.

\begin{tabular}{lc}
\hline \hline Technique & $T_{c}$ \\
\hline$M(T)$ at $0.001 \mathrm{~T}$ & $292 \mathrm{~K} \pm 2.5$ \\
$M(T)$ at $0.05 \mathrm{~T}$ (maximum of $d M / d T$ ) & $291 \mathrm{~K} \pm 2.5$ \\
$M(T)$ at $0.05 \mathrm{~T}$ (extrapolation from inflection) & $299.5 \mathrm{~K} \pm 2.5$ \\
Specific heat maximum & $289 \mathrm{~K} \pm 2.5$ \\
$\left(-\Delta S_{\max }\right)$ extrapolation & $293.8 \mathrm{~K} \pm 2.5$ \\
$T_{\max }=T_{c}-\gamma \Delta T_{c}, \gamma=1 / 6$ & \\
$\chi$ maximum at zero field & $282 \mathrm{~K}$ \\
Arrott plot $M^{1 / \beta}$ vs $(H / M)^{1 / \gamma}$ & \\
$\beta=0.5 ; \gamma=1$ & $302 \mathrm{~K}$ \\
$\beta=0.4 ; \gamma=4 / 3$ & $287.6 \mathrm{~K}$ \\
\hline \hline
\end{tabular}

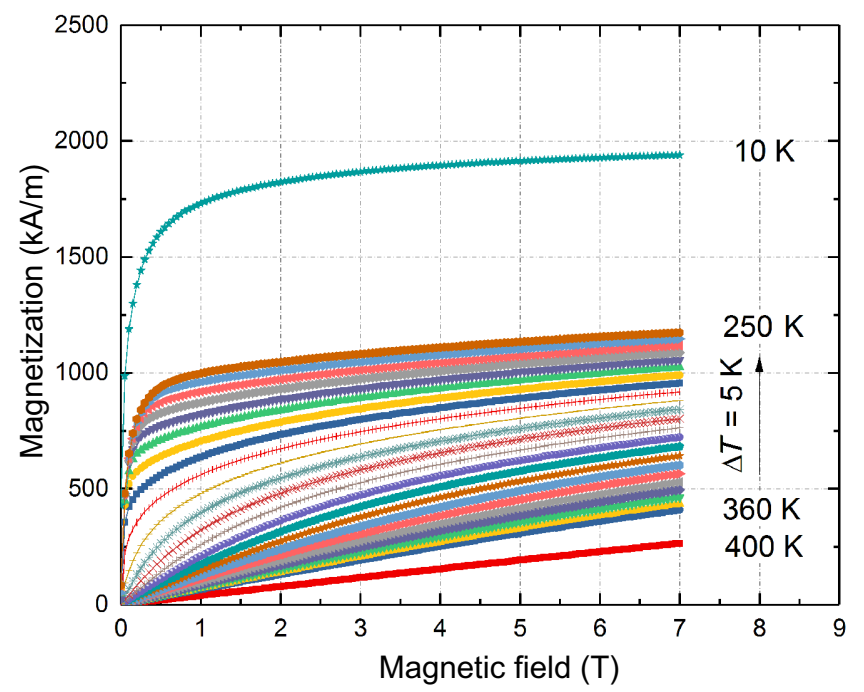

FIG. 7. Isothermal curves of the Gd free-standing film measured from 400 to $10 \mathrm{~K}$.

in Fig. 7. The $M(H)$ curve at $400 \mathrm{~K}$ clearly shows paramagnetic behavior. At $T<T_{c}$ the ferromagnetic ordering is apparent. Magnetic hysteresis loops measured in the ordered state show a soft magnetic behavior with a coercive field of $3.8 \mathrm{mT}$ at $200 \mathrm{~K}$ (full hysteresis cycles measurements are not shown here). As a further check, we used an Arrott plot (AP) method to extrapolate $T_{c}$ from the set of isothermal magnetization curves shown in Fig. 7. Figure 8 shows the standard AP (i.e., $M^{1 / \beta}$ versus $(H / M)^{1 / \gamma}$ with $\beta=0.5$ and $\left.\gamma=1\right)$ in the main frame, and a modified Arrott-Noakes plot (i.e., $M^{1 / \beta}$ versus $(H / M)^{1 / \gamma}$ with $\beta=0.4$ and $\gamma=4 / 3$ ) in the inset [28]. From the former, we get $T_{c}=302 \mathrm{~K}$ in agreement with the value

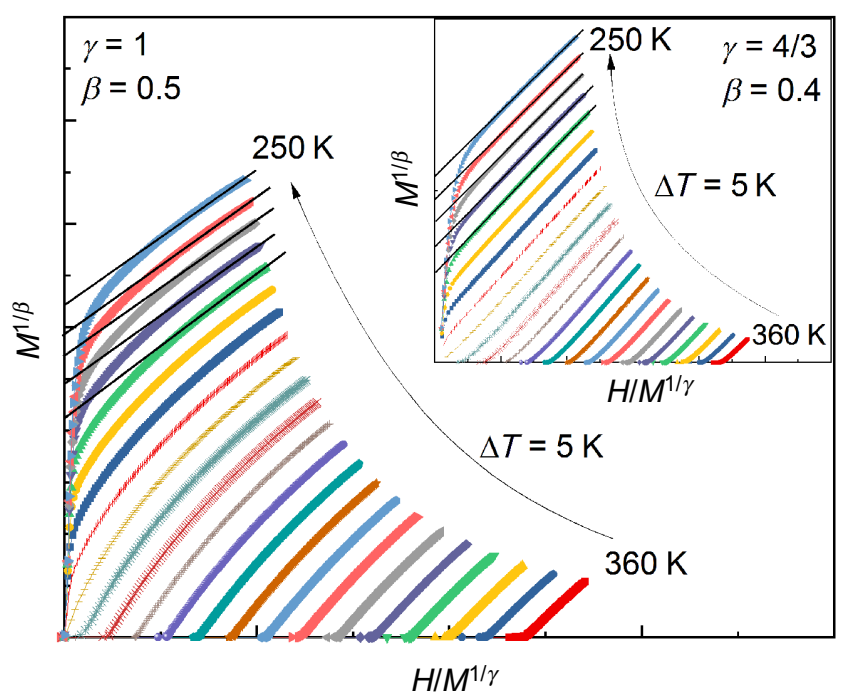

FIG. 8. Main frame: standard AP with $\beta=0.5$ and $\gamma=1$. Inset: modified Arrott-Noakes plot with $\beta=0.4$ and $\gamma=4 / 3$. 
worked out with the $M(T)$ inflection point method, while the latter gives $T_{c}=287.6 \mathrm{~K}$, a slightly underestimated value. The reliability of Arrott-Noakes equation of state, and its variants as a tool of material properties investigation has been largely addressed in the literature (see [29,30] and more in general [31] and references therein). What matters here is that the AP extrapolated values confirm that the free-standing film $T_{c}$ lays in the interval expected for high-purity bulk Gd.

\section{Entropy change}

The magnetic entropy change has been determined using the Maxwell relation [32]:

$$
\Delta S=\mu_{0} \int_{H_{i}}^{H_{f}}\left(\frac{\partial M}{\partial T}\right)_{H} d H,
$$

from the set of isotherms shown in Fig. 7, using the density bulk $\mathrm{Gd}$, namely $7.9 \times 10^{3} \mathrm{~kg} \mathrm{~m}^{-3}$, we work out the entropy change of the free-standing film for different $H_{f}$ values as shown in Fig. 10. The maximum entropy changes are $\left(-\Delta S_{\max }\right)=2.7,5.9,8.3$, and $10.5 \mathrm{~J} \mathrm{~kg}^{-1} \mathrm{~K}^{-1}$ at $H_{f}=1,3,5$, and $7 \mathrm{~T}$, respectively.

Notwithstanding the extreme sensitivity of such extrapolations to magnetization and mass uncertainties (i.e., $1 \%$ error on magnetic measurements may lead up to $10 \%$ error in entropy change [33]), the measured $\left(-\Delta S_{\max }\right)$ are in good agreement with the reported values for polycrystalline Gd (see [7,34] and references therein).

In addition to being one of the key functional properties for energy-conversion applications, the entropy change in the temperature interval surrounding $T_{c}$ can be used as a further tool to assess the quality of the samples. The temperature $T_{\max }$ corresponding to $\left(-\Delta S_{\max }\right)$ coincides with Curie point for pure Gd single crystals and may shift a few degrees below $T_{c}$ in polycrystals depending on their purity. Our measurements confirm this trend with $T_{\max }=$ $293 \mathrm{~K}$, as well as with the maximum entropy change following the expected mean-field power law $\left(-\Delta S_{\max }\right) \propto$ $H^{2 / 3}$ (Ref. [35]). Magnetic properties of single crystalline gadolinium were reported in the literature (for example, $[11,26])$.

Magnetic properties of the free-standing film presented here can be assessed against some reference results from the literature. In Fig. 9, the field dependence of the maximum entropy change $\left(-\Delta S_{\max }\right)$ of the Gd sample discussed here is compared with the properties of a cold rolled and annealed $36 \mu \mathrm{m}$ ribbon [20], and of a single crystal from the literature [11]. In the same figure, we show the properties of a $17 \mu \mathrm{m}$ Gd free-standing film, with tungsten capping layers, we deposited at higher temperature (i.e., substrate temperature of approximately $666 \mathrm{~K}$ ). It is worth noting that a wise choice of the deposition temperature and of the capping layer can allow MC properties closer to

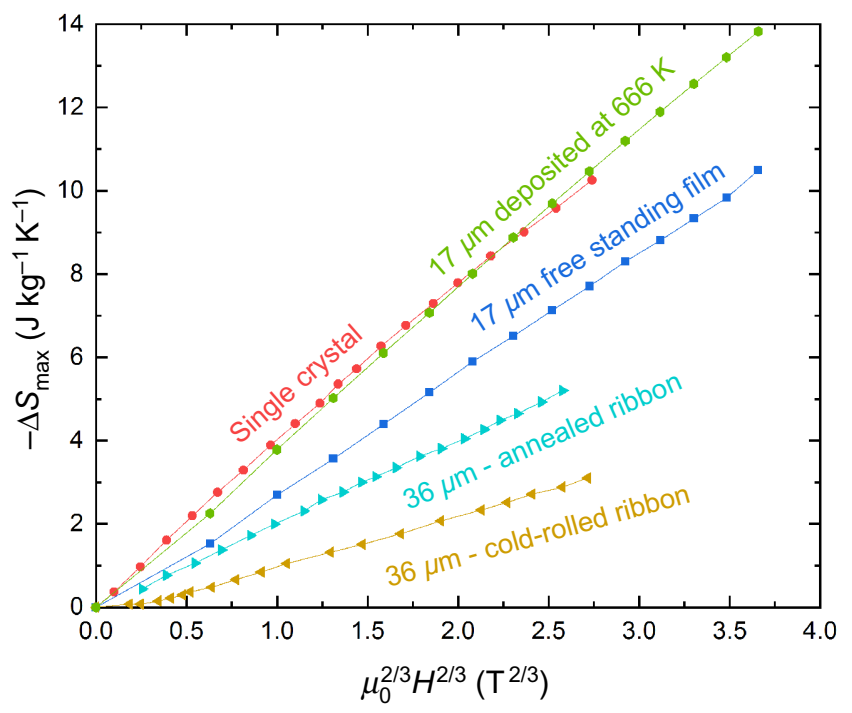

FIG. 9. Plots of $\left(-\Delta S_{\max }\right)$ against $H^{2 / 3}$ for the free-standing flexible $\mathrm{Ta} / \mathrm{Gd} / \mathrm{Ta}$ film presented here (blue), of a free-standing $\mathrm{W} / \mathrm{Gd} / \mathrm{W}$ film we deposited at higher temperature $(666 \mathrm{~K})$, using the same technique (green). In order to compare the entropy change with some results from the literature, the data of singlecrystal bulk Gd (red) from Ref. [11], of a cold-rolled (yellow), and an of an annealed after cold-rolling (cyan) ribbons [20] are also shown.

those of a Gd single crystal to be obtained. Nonetheless, as we discuss in what follows, here surface quality and flexibility are as relevant as $\left(-\Delta S_{\max }\right)$. As the sample showing a better MC behavior is less flexible due to the tungsten capping layer and the higher depositing temperature, we focus the present study on the film showing the best compromise between these three functional properties. A full discussion of the use of different capping layers and of the role of the deposition temperature would be beyond the scope of this work and will be presented elsewhere.

In order to gather further information on the presence of impurities and of defects in the free-standing film, we follow [11], describing the experimental $\Delta S(T)$ curves within Landau's approach. Purity and crystallization defects are expected to induce a distribution of $T_{c}$ of width $\Delta T_{c}$ that can be described through the following expression $\left(-\Delta S_{\max }\right)=A H^{2 / 3}+B$, where $A=\alpha /(4 b)^{2 / 3}$ and $B=-\alpha^{2} \Delta T_{C} /(18 b)$, material intrinsic parameters related to the Landau expansion coefficients. Temperature corresponding to the entropy maximum is estimated using the expression $T_{\max }=T_{c}-\gamma \Delta T_{c}$, where $\gamma$ is a coefficient dependent on the shape of the $T_{c}$ distribution. From this analysis, we get $T_{c}=293.8 \mathrm{~K}$ and $\Delta T_{c} \sim 4.7 \mathrm{~K}$, in good agreement with the values reported for bulk polycrystalline Gd with 99.7 at.\% (Ref. [11]). This $\Delta T_{c}$ corresponds to about one third the value we found on $3 \mu \mathrm{m} \mathrm{Gd} \mathrm{films}$ grown on silicon substrate with tungsten capping layers [14]. The narrower free-standing film $T_{c}$ distribution 


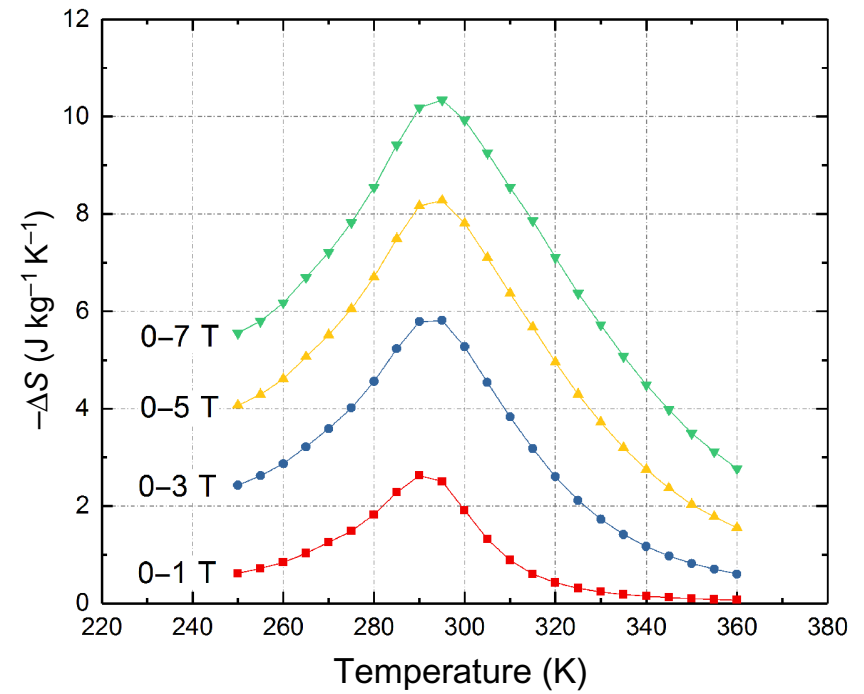

FIG. 10. Entropy changes of the free-standing film measured over different magnetic field ranges $0-H_{f}$ with $H_{f}=1,3,5$, and $7 \mathrm{~T}$.

suggests a reduced role of surface defects possibly due to the buffer, the capping layer, and to the increased thickness.

\section{Magnetic properties under strain}

Flexibility can be a key property allowing the film to operate under different strain conditions, and to make it compliant to the shape of curved surfaces without modifying its functional properties. To check the stability of the magnetic properties of Gd films under strain, we measured the magnetic AC susceptibility as a function of temperature, and the thermal magnetization curves $M(T)$, under bending comparing them with the behavior of the undeformed sample (as shown in Figs. 11 and 12). To carry out this comparison, the film has been bonded over a cylindrical support of diameter $d=2.17 \mathrm{~mm}$ (i.e., a radius of curvature $r=1.085 \mathrm{~mm}$ ), as schematically shown in the inset of Fig. 12. In this way, given the film thickness $h=17 \mu \mathrm{m}$, a tensile strain $\varepsilon=h / d=0.0078$ (i.e., a $0.78 \%$ strain) is applied on the convex side and a compressive strain $\varepsilon=-0.0078$ (i.e., a $-0.78 \%$ strain) is applied on the other. Magnetic measurements have been performed by applying the field along the cylinder axis.

Under the aforementioned bending conditions, neither the thermomagnetic curves nor the AC susceptibilities show any relevant change when measured under strain as apparent form Fig. 11 (main frame and inset, respectively).

Nonetheless, a slight departure of the measurement under strain from the unstrained values is apparent in the $M(T)$ curve measured under $0.05 \mathrm{~T}$, as well as in the susceptibility measurement, at temperatures below 250 $\mathrm{K}$. As this is the range of temperature where Gd undergoes a spin reorientation transition (i.e., a change in easy

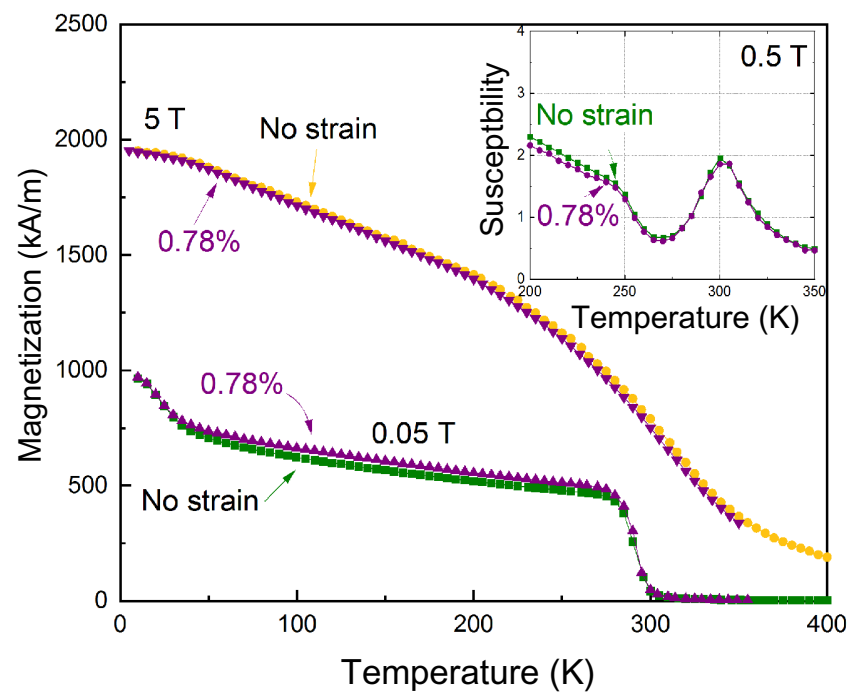

FIG. 11. The $M(T)$ curves before and after bending under 0.05 and $5 \mathrm{~T}$. The inset shows susceptibility measured at $0.5 \mathrm{mT} \mathrm{AC}$ magnetic field with a frequency of $250 \mathrm{~Hz}$ and DC magnetic field of $0.5 \mathrm{~T}$.

magnetization configuration) [36,37], the observed difference suggests a weak coupling between strain and the aforementioned transition [38].

Our bending setup submits the film faces to opposite applied stresses, compressive on the surface in contact with the cylinder, tensile on the other. This offers the opportunity to check the influence of the observed difference in

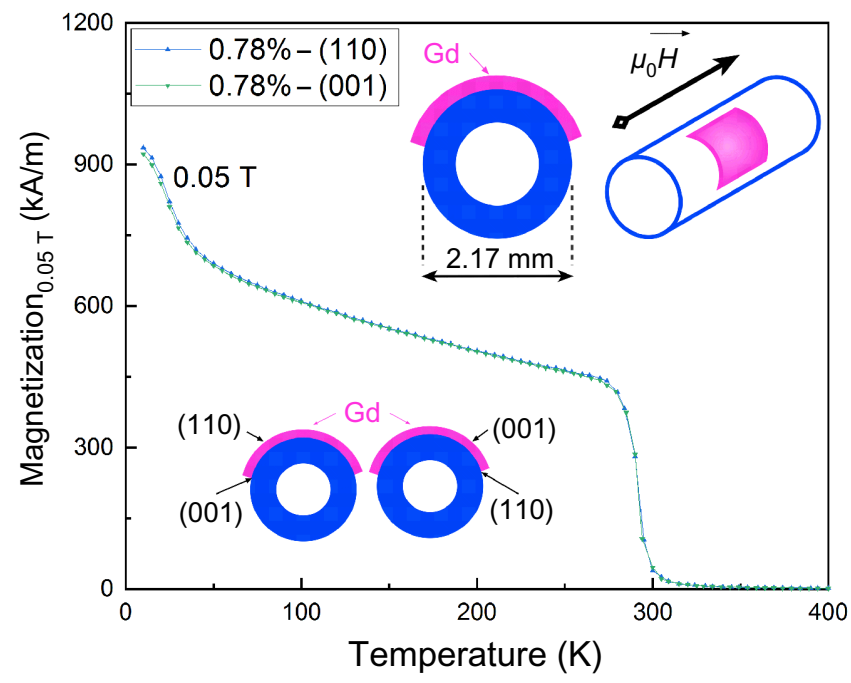

FIG. 12. The $M(T)$ curves of the free-standing film measured under two strain configurations, one where the (110) textured face is expanded and (001) face is compressed (the left draw on the bottom of the frame, blue upward triangles), the other where the (001) textured face is expanded and the (110) face is compressed (the right draw on the bottom of the frame, green downward triangles). 
texturing between the above mentioned top and bottom film surfaces on its magnetic properties under strain. To do that, we measured thermomagnetic curves under two different strain configurations: one with the bottom film surface bonded on the cylinder side (i.e., with the (001) textured surface under compressive stress and the (110) one under tensile stress), the other by flipping the film on the opposite side (see Fig. 12 inset). The two measurements are compared in Fig. 12 and are in good agreement showing that the difference in texturing between the two surfaces does not affect the overall magnetic behavior of the film.

\section{DISCUSSION AND CONCLUSIONS}

A variety of figures of merit to assess the properties of MC materials have been proposed (see, for instance, $[39,40]$ and references therein), however it is quite clear that the best MC material cannot be defined independently of the working principle of the device using it.

Since Barclay and Steyert's 1982 patent [41], active magnetic regeneration (AMR) has been the most used concept in $\mathrm{MC}$ refrigerator and heat pump prototypes. The main goal is widening the device temperature span using the $\mathrm{MC}$ material as a refrigerant and as a heat regenerator at the same time [42]. Temperature across the regenerator varies from that of the cold end to that of the hot end. Heat is exchanged through a fluid that has to be pumped through the MC material requiring a design maximizing the exchange surface (e.g., laminations, porous materials, microchannels). In its simplest configuration, an AMR device requires a motor to magnetize or demagnetize by changing the relative position of the MC material and of the magnets, and a pump to move the heat exchange fluid $[4,34]$. To date, prototypes using regenerative cycles have made possible some outstanding results becoming a sort of gold standard of caloric devices $[43,44]$. However, notwithstanding its high thermodynamic efficiency, as a recent review paper makes clear [4], AMR can hardly be expected to deliver high power densities making this solution unsuitable when the mass of the caloric material has to be kept as low as possible.

Recently, flexible caloric films paved the way to an alternative paradigm. In the new generation of energy transducers, based on poly(vinylidene fluoride) (PVDF) electrocaloric and natural-rubber elastocaloric materials [45-48], regeneration is replaced by a cascade of devices, whereas heat transfer is dealt with through solid-solid direct contact, making heat exchange fluid and the pumps redundant.

Direct solid-solid heat contact puts forth high surface quality (i.e., low roughness) and active material flexibility (i.e., the possibility to conform to a surface through relatively moderate acting forces) as key features to attain unprecedented thermal management efficiencies [49,50], one of the main ways towards output power and efficiency enhancement of caloric devices [4].

When the thermal exchange at the interface is not the limiting factor, the frequency is limited by thermal conduction inside the material (i.e., the time $t$ heat needs to diffuse through the material). Thin film aspect ratio makes a cycling frequency increase of orders of magnitudes possible. This makes the heat transfer time proportional to the square of the film thickness $h$, namely $t \sim h^{2} / \alpha$ where $\alpha$ is the thermal diffusivity [51]. As the energy stored by the material $\mathcal{E}$ scales as its volume, $\mathcal{E} \sim h$, the device maximum power $P=\mathcal{E} / t$ scales as $\sim 1 / h$. Although microhard-magnet arrays films are already available [52], we know of no flexible-MC-film-based devices that have been presented so far.

In this respect, the properties of the Gd film presented here, in terms of thickness, flexibility, smoothness, and $\mathrm{MC}$ effect, represent a unique opportunity to pinpoint flexible MC films as an active substance for small-scale, high-throughput energy-conversion devices based on the paradigm, where high powers can be attained by increasing the working frequency through an improved management of the thermal exchange $[51,53]$.

Actually, designs where the active material moves between the hot and the cold end, exchanging heat through direct contact, have been used for thermal energy harvesting using bulk MC materials [24,25,54] and films [6,55]. In this case, self-actuation is the key feature as the field source switches the caloric effect, and provides the acting force being the motor and the pump at the same time. This allows a pretty relevant design simplification, an improved overall efficiency due to the absence of the motor and pump energy consumption, and lets us foresee the development of small batteryless autonomous generators with possible applications to Internet of Things (IoT) devices and medical implants.

More specifically, the prototypes presented in Refs. [24,25] have been conceived in view of a possible miniaturization and, although micromagnets arrays have been available for more than a decade [2,52,56, 57], no flexible MC films or membrane, in the tens of micrometer thickness range, showing high-quality surface, and with good functional properties, have been reported to date.

Let us now consider a MC thermal harvesting device deploying the above-presented $\mathrm{Gd}$ free-standing film. Using a patterned hard magnetic film [2,52], it is possible to get magnetic fields well confined in space, in order to minimize the demagnetizing effect, and showing pretty steep gradients over small distances (e.g., a field change from 0 to about $1 \mathrm{~T}$ over less than $50 \mu \mathrm{m}$ displacement). Figure 13 shows a possible design: the active material (i.e., the Gd film, green line) moves between a patterned magnet on the top and the heat sink on the bottom. A wise balance between the magnetic force due to the 


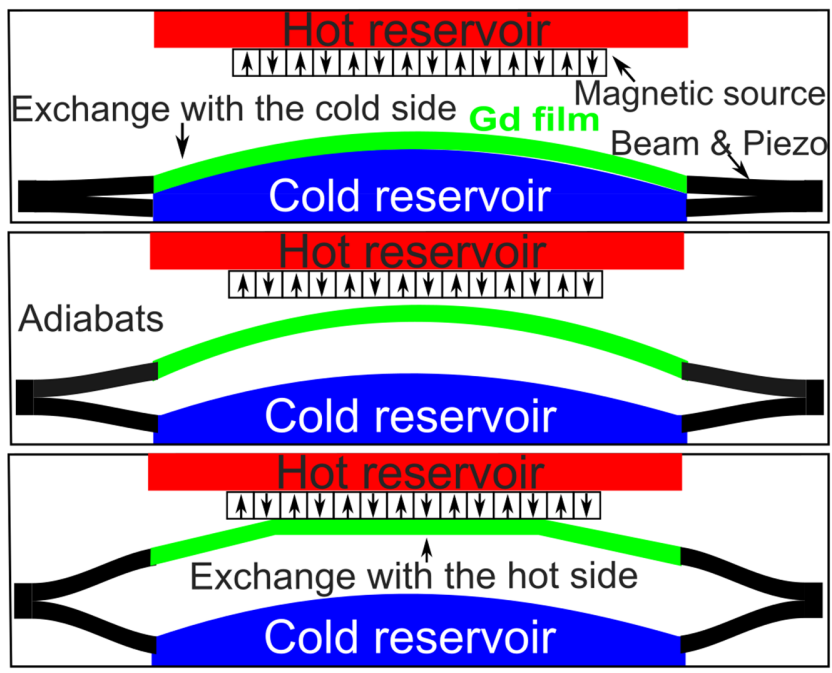

FIG. 13. Schematic drawing of the device. The adiabatic magnetization and demagnetization (adiabats) are represented by the central frame (the MC material moving upward to magnetize and downward to demagnetize), the top and bottom drawings show the constant applied field and heat exchange transformations (cooling and heating, respectively). The schematic device drawing emphasizes the relevance of the Gd film flexibility in order to achieve an optimal thermal contact with the reservoirs.

field gradient and to the magnetization of the MC material, and the elastic recall force (here associated with a piezoelectric beam sketched in black) drives the moving part reaching a self-oscillating regime (for more details, see Ref. [24,25]). Considering the above-presented entropy change, and assuming an adiabatic temperature change of $2.4 \mathrm{~K}$ (high-purity Gd polycrystals shows $2.9 \mathrm{~K}$ [7]), we can estimate an upper bound for the generator power output using a finite-time thermodynamic approach [58]. The thermodynamic cycle associated with the example design is shown in Fig. 14. It is a four-stroke Brayton cycle, composed by two adiabats, where the field changes, and two transformations at constant field where the active material exchanges heat with the reservoirs.

The point with finite-time thermodynamics models is that power is extrapolated from the state variables (field, temperature, entropy) and from the thermal exchange constants describing the heat fluxes between the engine and the reservoirs [59-61]. The thermodynamic generalized force driving heat exchange between the active substance and the reservoirs is the temperature difference between the former and the latter $\Delta T_{\text {exch }}$, as shown in Fig. 14, where a Brayton cycle is shown in the temperature-entropy $(T, S)$ plane.

Through the thermal-switch model we recently presented [62], and relying on the Gd film surface quality, we can obtain a reasonable estimate of the thermal conduction constants. Using similar working conditions to those studied in Ref. [25], namely a single stage device

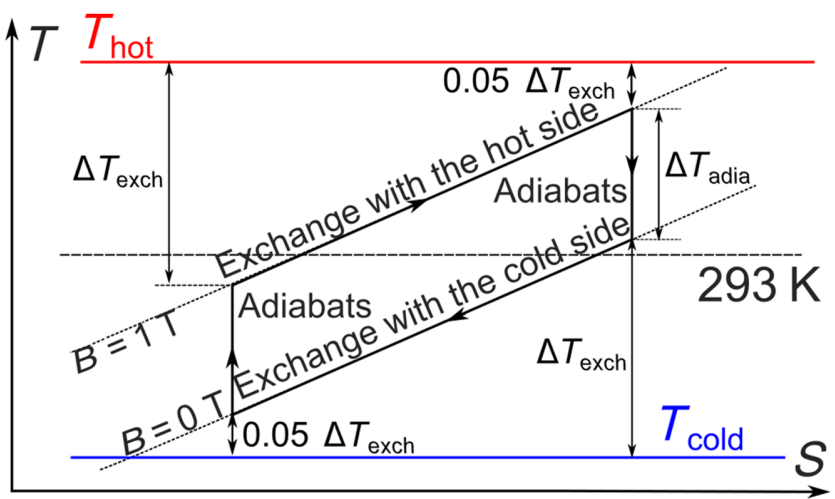

FIG. 14. A Brayton thermodynamic cycle represented in the temperature-entropy plane. The cycle is composed by the following four transformations: adiabatic magnetization, exchange with the hot side under constant applied field, adiabatic demagnetization, and exchange with the heat sink at constant applied field. The cycle shown here has been used to get the power estimation discussed in the main text. The oscillation period has been designed in order to get the adiabatic switch when heat exchange has reduced the temperature difference between the magnetic material and the reservoir ( $\Delta T_{\text {exch }}$ in the figure) of $95 \%$ with respect to its initial value.

cycling over a fixed temperature difference between the hot and cold reservoir of $\Delta T_{\text {res }}=10 \mathrm{~K}$ (Fig. 13), we get a magnetic power of $1.1 \mathrm{~W} \mathrm{~g}^{-1}\left(16 \mathrm{~mW} \mathrm{~cm}^{-2}\right)$, corresponding to a frequency of $100 \mathrm{~Hz}$. Considering a final conversion ratio between magnetic and electrical energy of $10^{-3}$ as in Ref. [25], we get an electrical power output of $16 \mu \mathrm{W} \mathrm{cm}{ }^{-2}$, corresponding to more than four orders of magnitude higher than the value reported in Ref. [25], and fits pretty well the needs of the recent generation of wireless IoT sensors [63]. This is just a coarse estimation where the $10^{-3}$ factor takes into account the many loss phenomena an actual device has to tackle (e.g., eddy currents, heat leakages), and the efficiency of the final conversion step towards electrical power (e.g., piezoelectric, capacitive, inductive). Nonetheless, the result points at the actual technological opportunities opened by the development of free-standing MC films where flexibility and absence of substrate are the key issues towards a fast and efficient heat transfer.

Summarizing, we presented a method to produce thick Gd free-standing polycrystalline films showing magnetic properties (i.e., saturation magnetization, Curie temperature) close to those of high-purity bulk Gd. The film is flexible, smooth, and its MC properties are stable under strain. Using a thermal switch model we presented elsewhere [62], we show that the availability of freestanding flexible MC films represents a key result towards the next generation of compact MC energy-conversion devices. 


\section{ACKNOWLEDGMENTS}

This research has received funding from the French National Research Agency under the project HiPerTherMag (ANR-18-CE05-0019). The authors acknowledge the staff (and, in particular, David Hrabovsky and Yanling Li) of the MPBT (physical properties, low temperature) platform of Sorbonne Université for their support, Ian Vickridge for RBS measurements at SAFIR accelerator, and Hervé Cruguel for AFM measurements at the AFMAV platform. We would also wish to thank Nora Dempsey, Erika Fontana, and Thibaut Devilliers for helpful discussions.

[1] G. Scheunert, O. Heinonen, R. Hardeman, A. Lapicki, M. Gubbins, and R. M. Bowman, A review of high magnetic moment thin films for microscale and nanotechnology applications, Appl. Phys. Rev. 3, 011301 (2016).

[2] N. M. Dempsey, A. Walther, F. May, D. Givord, K. Khlopkov, and O. Gutfleisch, High performance hard magnetic NdFeB thick films for integration into micro-electromechanical systems, Appl. Phys. Lett. 90, 092509 (2007).

[3] N. Jackson, F. J. Pedrosa, A. Bollero, A. Mathewson, and O. Z. Olszewski, Integration of thick-film permanent magnets for mems applications, J. Microelectromech. Syst. 25, 716 (2016).

[4] A. Kitanovski, Energy applications of magnetocaloric materials, Adv. Energy Mater. 10, 1903741 (2020).

[5] T. Tsukamoto, M. Esashi, and S. Tanaka, Magnetocaloric cooling of a thermally-isolated microstructure, J. Micromech. Microeng. 22, 094008 (2012).

[6] M. Gueltig, F. Wendler, H. Ossmer, M. Ohtsuka, H. Miki, T. Takagi, and M. Kohl, High-performance thermomagnetic generators based on heusler alloy films, Adv. Energy Mater. 7, 1601879 (2017).

[7] J. Lyubina, Magnetocaloric materials for energy efficient cooling, J. Phys. D: Appl. Phys. 50, 053002 (2017).

[8] F. An, K. Qu, G. Zhong, Y. Dong, W. Ming, M. Zi, Z. Liu, Y. Wang, B. Qi, Z. Ding, et al., Highly flexible and twistable freestanding single crystalline magnetite film with robust magnetism, Adv. Funct. Mater. 30, 2003495 (2020).

[9] H.-R. Lim, H. S. Kim, R. Qazi, Y.-T. Kwon, J.-W. Jeong, and W.-H. Yeo, Advanced soft materials, sensor integrations, and applications of wearable flexible hybrid electronics in healthcare, energy, and environment, Adv. Mater. 32, 1901924 (2020).

[10] K. P. Belov, R. Levitin, and S. Nikitin, Reviews of topical problems: Ferromagnetism and antiferromagnetism of rareearth metals, Sov. Phys. Usp. 7, 179 (1964).

[11] J. Lyubina, M. D. Kuz'min, K. Nenkov, O. Gutfleisch, M. Richter, D. L. Schlagel, T. A. Lograsso, and K. A. Gschneidner, Jr., Magnetic field dependence of the maximum magnetic entropy change, Phys. Rev. B 83, 012403 (2011).

[12] G. Scheunert, W. R. Hendren, C. Ward, and R. M. Bowman, Magnetization of $2.6 \mathrm{~T}$ in gadolinium thin films, Appl. Phys. Lett. 101, 142407 (2012).
[13] T. F. Ambrose, Magnetic write head with thermoelectric cooling device, U.S. Patent No. 8,031,435 (2011).

[14] N. B. Doan, L. Becerra, N. Casaretto, J.-E. Duvauchelle, M. Marangolo, S. Ahmim, M. Almanza, and M. LoBue, Magnetocaloric gadolinium thick films for energy harvesting applications, AIP Adv. 10, 035110 (2020).

[15] L. Helmich, M. Bartke, N. Teichert, B. Schleicher, S. Fähler, and A. Hätten, Gadolinium thin films as benchmark for magneto-caloric thin films, AIP Adv. 7, 056429 (2017).

[16] G. Scheunert, C. Ward, W. R. Hendren, A. A. Lapicki, R. Hardeman, M. Mooney, M. Gubbins, and R. M. Bowman, Influence of strain and polycrystalline ordering on magnetic properties of high moment rare earth metals and alloys, J. Phys. D: Appl. Phys. 47, 415005 (2014).

[17] W. Dun-hui, H. Zhi-da, H. Song-ling, P. Kun, W. Yi, Z. Wen-qin, G. Ben-xi, and D. You-wei, The low-field magnetic entropy change in melt-spun and annealed Gd ribbons, Physica B: Condens. Matter 352, 185 (2004).

[18] A. Svalov, S. Andreev, A. Arkhipov, E. Kudyukov, D. Neznakhin, A. Larrañaga, and G. Kurlyandskaya, in Journal of Physics: Conference Series, Vol. 1389 (IOP Publishing, 2019), p. 012100.

[19] A. Arkhipov, S. Andreev, D. Neznakhin, A. Tebenkov, A. Larrañaga, G. Kurlyandskaya, and A. Svalov, in AIP Conference Proceedings, Vol. 2313 (AIP Publishing LLC, 2020), p. 030036.

[20] S. V. Taskaev, V. D. Buchelnikov, A. P. Pellenen, M. D. Kuz'min, K. P. Skokov, D. Y. Karpenkov, D. S. Bataev, and O. Gutfleisch, Influence of thermal treatment on magnetocaloric properties of Gd cold rolled ribbons, J. Appl. Phys. 113, 17A933 (2013).

[21] S. Taskaev, K. Skokov, V. Khovaylo, V. Buchelnikov, A. Pellenen, D. Karpenkov, M. Ulyanov, D. Bataev, A. Usenko, M. Lyange, et al., Effect of severe plastic deformation on the specific heat and magnetic properties of cold rolled Gd sheets, J. Appl. Phys. 117, 123914 (2015).

[22] S. Taskaev, K. Skokov, V. Khovaylo, M. Ulyanov, D. Bataev, D. Karpenkov, I. Radulov, A. Dyakonov, and O. Gutfleisch, Magnetocaloric effect in cold rolled foils of $\operatorname{Gd}_{100-x} \operatorname{In}_{x}(x=0,1,3)$, J. Magn. Magn. Mater. 459, 46 (2018).

[23] K. Shinde, B. Sinha, S. Oh, H. Kim, H. Ha, S. Baik, K. Chung, D. Kim, and S. Jeong, Fabrication of gd films by vacuum evaporation and its magnetocaloric properties, J. Magn. Magn. Mater. 374, 144 (2015).

[24] S. Ahmim, M. Almanza, A. Pasko, F. Mazaleyrat, and M. Lobue, Thermal energy harvesting system based on magnetocaloric materials, Eur. Phys. J. Appl. Phys. 85, 10902 (2019).

[25] S. Ahmim, M. Almanza, V. Loyau, A. Pasko, F. Mazaleyrat, and M. LoBue, A thermal energy harvester using LaFeSi magnetocaloric materials, IEEE Trans. Magn. 57, 1 (2021).

[26] S. Y. Dan'kov, A. M. Tishin, V. K. Pecharsky, and K. A. Gschneidner, Magnetic phase transitions and the magnetothermal properties of gadolinium, Phys. Rev. B 57, 3478 (1998).

[27] F. Fiorillo, Characterization and Measurement of Magnetic Materials (Academic Press, Amsterdam, 2004). 
[28] A. Arrott and J. E. Noakes, Approximate Equation of State for Nickel near its Critical Temperature, Phys. Rev. Lett. 19, 786 (1967).

[29] J. S. Kouvel and M. E. Fisher, Detailed magnetic behavior of nickel near its Curie point, Phys. Rev. 136, A1626 (1964).

[30] C. W. Miller, D. V. Williams, N. S. Bingham, and H. Srikanth, Magnetocaloric effect in $\mathrm{Gd} / \mathrm{W}$ thin film heterostructures, J. Appl. Phys. 107, 09A903 (2010).

[31] V. Franco, J. Blázquez, B. Ingale, and A. Conde, The magnetocaloric effect and magnetic refrigeration near room temperature: Materials and models, Annu. Rev. Mater. Res. 42, 305 (2012).

[32] A. Smith, C. R. Bahl, R. Bjørk, K. Engelbrecht, K. K. Nielsen, and N. Pryds, Materials challenges for high performance magnetocaloric refrigeration devices, Adv. Energy Mater. 2, 1288 (2012).

[33] V. K. Pecharsky and K. A. Gschneidner, Magnetocaloric effect from indirect measurements: Magnetization and heat capacity, J. Appl. Phys. 86, 565 (1999).

[34] J. Lyubina, in Novel Functional Magnetic Materials (Springer International Publishing, Switzerland, 2016), p. 115.

[35] H. Oesterreicher and F. Parker, Magnetic cooling near Curie temperatures above 300 K, J. Appl. Phys. 55, 4334 (1984).

[36] W. Corner, W. Roe, and K. Taylor, The magnetocrystalline anisotropy of gadolinium, Proc. Phys. Soc. 80, 927 (1962).

[37] M. Colarieti-Tosti, T. Burkert, O. Eriksson, L. Nordström, and M. S. Brooks, Theory of the temperature dependence of the easy axis of magnetization in hcp Gd, Phys. Rev. B 72, 094423 (2005).

[38] A. V. Andrianov and E. Bauer, Magnetocrystalline phase diagram of Gd: Probably a novel phase boundary, Europhys. Lett. 102, 17011 (2013).

[39] K. G. Sandeman, Magnetocaloric materials: The search for new systems, Scr. Mater. 67, 566 (2012), viewpoint Set No. 51: Magnetic Materials for Energy.

[40] I. Niknia, P. Trevizoli, T. Christiaanse, P. Govindappa, R. Teyber, and A. Rowe, Material screening metrics and optimal performance of an active magnetic regenerator, J. Appl. Phys. 121, 064902 (2017).

[41] J. A. Barclay and W. A. Steyert, Active magnetic regenerator, U.S. Patent No. 4,332,135 (1982).

[42] M.-A. Richard, A. Rowe, and R. Chahine, Magnetic refrigeration: Single and multimaterial active magnetic regenerator experiments, J. Appl. Phys. 95, 2146 (2004).

[43] J. Tušek, K. Engelbrecht, D. Eriksen, S. Dallolio, J. Tušek, and N. Pryds, A regenerative elastocaloric heat pump, Nat. Energy 1, 16134 (2016).

[44] U. Plaznik, M. Vrabelj, Z. Kutnjak, B. Malič, B. Rožič, A. Poredoš, and A. Kitanovski, Numerical modelling and experimental validation of a regenerative electrocaloric cooler, Int. J. Refrig. 98, 139 (2019).

[45] R. Ma, Z. Zhang, K. Tong, D. Huber, R. Kornbluh, Y. S. $\mathrm{Ju}$, and Q. Pei, Highly efficient electrocaloric cooling with electrostatic actuation, Science 357, 1130 (2017).

[46] Y. Meng, Z. Zhang, H. Wu, R. Wu, J. Wu, H. Wang, and Q. Pei, A cascade electrocaloric cooling device for large temperature lift, Nat. Energy 5, 996 (2020).

[47] Y. Bo, Q. Zhang, H. Cui, M. Wang, C. Zhang, W. He, X. Fan, Y. Lv, X. Fu, J. Liang, Y. Huang, R. Ma, and
Y. Chen, Electrostatic actuating double-unit electrocaloric cooling device with high efficiency, Adv. Energy Mater. 11, 2003771 (2021).

[48] F. Greibich, R. Schwödiauer, G. Mao, D. Wirthl, M. Drack, R. Baumgartner, A. Kogler, J. Stadlbauer, S. Bauer, N. Arnold, and M. Kaltenbrunner, Elastocaloric heat pump with specific cooling power of $20.9 \mathrm{~W} \mathrm{~g}^{-1}$ exploiting snap-through instability and strain-induced crystallization, Nat. Energy 6, 260 (2021).

[49] H. Keum, M. Seong, S. Sinha, and S. Kim, Electrostatically driven collapsible $\mathrm{Au}$ thin films assembled using transfer printing for thermal switching, Appl. Phys. Lett. 100, 211904 (2012).

[50] M. Almanza, A. Pasko, F. Mazaleyrat, and M. LoBue, First- versus second-order magnetocaloric material for thermomagnetic energy conversion, IEEE Trans. Magn. 53, 1 (2017).

[51] J. P. Feser and J. Ravichandran, More power to pyroelectrics, Nat. Mater. 17, 385 (2018).

[52] D. Le Roy, G. Shaw, R. Haettel, K. Hasselbach, F. DumasBouchiat, D. Givord, and N. M. Dempsey, Fabrication and characterization of polymer membranes with integrated arrays of high performance micro-magnets, Mater. Today Commun. 6, 50 (2016).

[53] K. Klinar and A. Kitanovski, Thermal control elements for caloric energy conversion, Renew. Sustain. Energy Rev. 118, 109571 (2020).

[54] M. Ujihara, G. Carman, and D. Lee, Thermal energy harvesting device using ferromagnetic materials, Appl. Phys. Lett. 91, 093508 (2007).

[55] J. Joseph, M. Ohtsuka, H. Miki, and M. Kohl, Upscaling of thermomagnetic generators based on heusler alloy films, Joule 4, 2718 (2020).

[56] A. Walther, D. Givord, N. Dempsey, K. Khlopkov, and O. Gutfleisch, Structural, magnetic, and mechanical properties of $5 \mu \mathrm{m}$ thick SmCo films suitable for use in microelectromechanical systems, J. Appl. Phys. 103, 043911 (2008).

[57] F. Dumas-Bouchiat, L.-F. Zanini, M. Kustov, N. Dempsey, R. Grechishkin, K. Hasselbach, J.-C. Orlianges, C. Champeaux, A. Catherinot, and D. Givord, Thermomagnetically patterned micromagnets, Appl. Phys. Lett. 96, 102511 (2010).

[58] M. Almanza, A. Pasko, F. Mazaleyrat, and M. LoBue, Numerical study of thermomagnetic cycle, J. Magn. Magn. Mater. 426, 64 (2017).

[59] F. Curzon and B. Ahlborn, Efficiency of a Carnot engine at maximum power output, Am. J. Phys. 43, 22 (1975).

[60] B. Andresen, P. Salamon, and R. S. Berry, Thermodynamics in finite time, Phys. Today 37, No. 9, 62 (1984).

[61] C. Van den Broeck, Thermodynamic Efficiency at Maximum Power, Phys. Rev. Lett. 95, 190602 (2005).

[62] M. Almanza, L. Depreux, F. Parrain, and M. LoBue, Electrostatically actuated thermal switch device for caloric film, Appl. Phys. Lett. 112, 083901 (2018).

[63] D. Newell and M. Duffy, Review of power conversion and energy management for low-power, low-voltage energy harvesting powered wireless sensors, IEEE Trans. Power Electron. 34, 9794 (2019). 